\title{
Use of Bisphosphonates, Calcium and Vitamin D for Bone Demineralization in Patients with Human Immunodeficiency Virus/Acquired Immune Deficiency Syndrome: A Systematic Review and Meta-Analysis of Clinical Trials
}

\author{
Vinícius Magno da Rocha ${ }^{1,2}$, Mariana Balardino Bogado Faria², Francisco de Assis dos Reis Júnior ${ }^{3}$, \\ Carla Ormundo Gonçalves Ximenes Lima ${ }^{4}$, Rossano Kepler Alvim Fiorelli ${ }^{1}$, Keila Mara Cassiano ${ }^{5}$ \\ ${ }^{1}$ Department of General and Specialized Surgery, Medical School, Federal University of the State of Rio de Janeiro, RJ; \\ ${ }^{2}$ Department of Orthopedics and Traumatology, Gaffrée and Guinle University Hospital, RJ; \\ ${ }^{3}$ Dertment of Orthopedics and Traumatology, Central Brazilian Army Hospital, RJ; \\ ${ }^{4}$ Department of Clinical Pathology, National Institute of Traumatology and Orthopedics, RJ; \\ ${ }^{5}$ Department of Statistics, Institute of Mathematics, Federal Fluminense University, RJ, Brazil
}

Corresponding author

Vinícius Magno da Rocha

Department of General and Specialized

Surgery, Medical School, Federal University of the State of Rio de Janeiro, Rua

Desembargador Izidro, 18 - Room 912, Tijuca,

Rio de Janeiro, RJ 20521-160, Brazil

Tel: +55-21-2209-2194; +55-21-99700-7240

Fax: +55-21-2298-2194

E-mail: viniciusmagnodarocha@gmail.com

Received: April 21, 2020

Revised: July 6, 2020

Accepted: July 8, 2020
Copyright $@ 2020$ The Korean Society for Bone and Mineral Research

This is an Open Access article distributed under the terms of the Creative Commons Attribution Non-Commercial License (https://creativecommons.org/licenses/by-nc/4.0/) which permits unrestricted non-commercial use, distribution, and reproduction in any medium, provided the original work is properly cited.
Background: The present study performed a systematic review and meta-analysis of clinical trials using bisphosphonates for bone demineralization in human immunodeficiency virus (HIV) patients. Methods: A comprehensive literature search was performed from January 2004 to January 2020 considering the bone mineral density (BMD) of the lumbar spine (LS) as the main outcome. Out of 214 titles that met criteria, 9 studies fulfilled the selection criteria. Results: A total of 394 patients were identified, and they were allocated into 2 groups: the intervention group (200 patients), to whom a combination of alendronate or zoledronate with calcium and vitamin D was administered; and control group (194 patients), to whom only calcium and vitamin D was administered. Clinical profile and indicators of bone metabolism of the participants were evaluated regarding effect size, homogeneity, and consistency. No substantial heterogeneity between the groups was found for the baseline variables, and there was high consistency to the main outcome. The meta-analysis shows a significant difference in post-treatment BMD, favoring the intervention over the control treatment. The intervention improved LS density up to $0.227 \mathrm{~g} / \mathrm{cm}^{2}$, raising the average to the levels of general population. Adverse effects related to intervention were fever immediately after zoledronate administration and gastrointestinal complaints during alendronate usage. Other adverse effects were barely reported and poorly connected to intervention by studies' authors, despite all of them have been successfully resolved. Conclusions: This study provides evidence that BMD post-treatment is better in HIV patients who used bisphosphonates combined with calcium and vitamin D.

Key Words: Antiretroviral therapy, highly active $\cdot$ Bone demineralization, pathologic $\cdot$ Diphosphonates · HIV · Osteoporosis

\section{INTRODUCTION}

Advances in the treatment of acquired immune deficiency syndrome (AIDS) impacts directly on the demographic profile of the infection. Highly active antiretro- 
viral therapy (HAART) is increasing gradually life expectancy of people living with human immunodeficiency virus (HIV), allowing a better understanding of associations between the virus and the changes inherent to the aging process.[1-4]

Bone mineral density (BMD) reduction is a known finding related to aging that has been discussed for decades as the main pathophysiological drive of osteoporosis - a disease in which bone quality, density, and resistance are reduced, resulting in an increased risk of fractures.[5]

Osteoporosis is defined by a BMD reduction until 2.5 (or more) standard deviation (SD) below the average for the Caucasian population using dual energy $\mathrm{X}$-ray absorptiometry as a measurement tool. Likewise, the disease is also defined by the occurrence of a frailty fracture.[6]

Among HIV patients, osteoporosis is more prevalent than in the general population, despite the age group and the use of HAART, which highlights the virus as an isolated risk factor for bone demineralization.[2,3] Furthermore, additional decreases in BMD were demonstrated after starting HAART, establishing pathways for bone demineralization different of those correlated to virus itself.[7]

As number of people over 50 living with HIV has been growing markedly since HAART introduction, the deleterious effects of HIV and its treatment on bone metabolism is calling the attention of researches and health managers due to the greater risk of frailty fractures in a group of patients with many comorbidities and high rates of post-surgical complications were expected.[8-11]

Driven by this warning, many studies have been conducted in recent decades to clarify the risk of frailty fractures, as well as to identify treatment strategies for bone demineralization in HIV patients.[12-19]

As the use of bisphosphonates, calcium, and vitamin $D$ is a widespread option against bone demineralization in the general population, and we performed a systematic review and meta-analysis about the effects of bisphosphonates, calcium and vitamin $D$ in bone demineralization of patients with HIV/AIDS.

\section{METHODS}

A systematic review and meta-analysis of the literature were performed according to the Cochrane Manual for Systematic Reviews of Interventions and Preferred Report- ing Items for Systematic Review and Meta-Analysis recommendations.[20,21]

An extensive literature search was conducted in electronic databases Medline, OVID, Cochrane Library, and EMBASE from January 2004 to January 2020, using the following descriptors: "HIV", "AIDS", "acquired immunodeficiency syndrome", "bisphosphonate", "vitamin D", "vitamin D3", "(25-hydroxyvitamin D)", "1,25-hydroxyvitamin D3 (25-hydroxyvitamin D3)", "cholecalciferol", "calcium (calcium)", "1,25-hydroxyvitamin D bone mineral density", "osteopenia", "osteoporosis", "fracture", "frailty, bone disease".

Only trials published in English were selected. An inclusion criterion was the use of bisphosphonates, vitamin D, and calcium on HIV patients against bone demineralization, regardless the dosage used. The use of any other drug to enhance BMD was an exclusion criterion for the trials. The main outcome considered in meta-analysis was the lumbar spine (LS) BMD evaluated by bone densitometry.

Other variables related to BMD were also included in this meta-analysis: patients' age, time of HIV infection, nadir of CD4+ T cells, CD4+ T cells count, body mass index (BMI), tobacco consumption, alcohol consumption, proportion of Caucasians, and LS T-score. Therefore, it is important to show that the intervention and control groups of the global meta-analysis sample were under the same comparison conditions, with no significant differences in baseline variables distribution. So, for BMD outcome and baselines variables, the meta-analysis groups were compared regarding effect size, homogeneity, and consistency. We used Cochrane's $Q$ and $I^{2}$ statistics to address heterogeneity and consistency of trials (significant if $P \leq 0.05$ in $Q$ and $I^{2}$ greater than $50 \%)$. Effect sizes were reported as odds ratios for qualitative factors or as standardized mean difference for continuous variables, and $95 \%$ confidence intervals.

Two reviewers, an orthopedist and an infectologist specialized in HIV/AIDS evaluated the search results. Titles and abstracts of trials were submitted for evaluation. Manuscripts were provided in full whenever the information was considered insufficient to meet the eligibility criteria. Data were collected independently by reviewers following a prototype database in an electronic spreadsheet developed by the authors for this purpose. The agreement between the evaluators in the construction of the database was assessed by Cohen's $\mathrm{K}$ coefficient. Divergent choices regarding the selection of a study were solved by consensus. For each tri- 


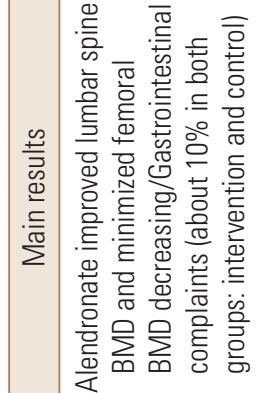

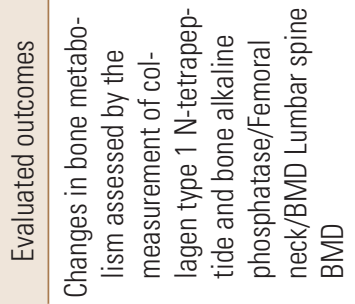

㐫 응

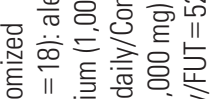

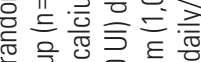

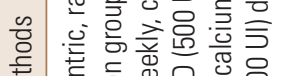

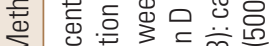

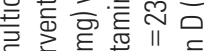

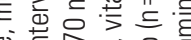

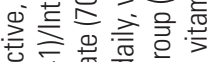

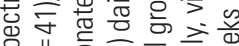

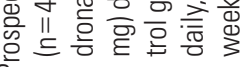

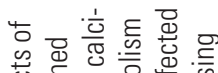

选

क के हो

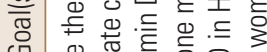

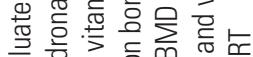

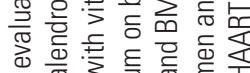

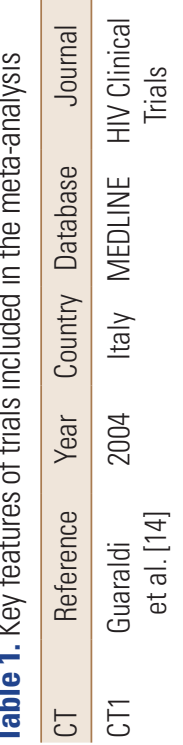

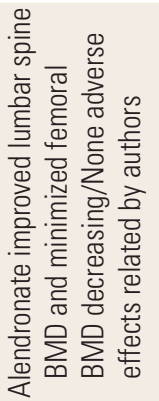

产 空

定总埌只

응응

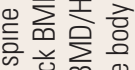

क⿺辶一兀

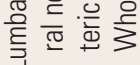

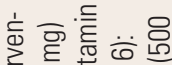

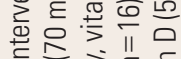

焉 离

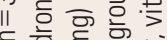

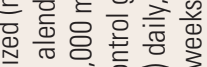

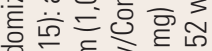

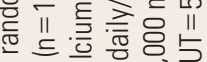

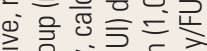

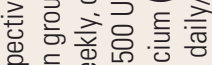

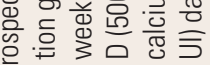

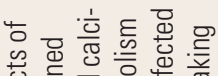

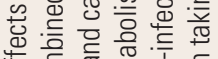

记

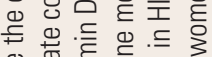

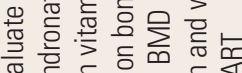

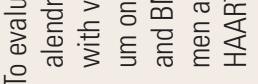

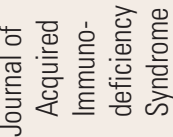

崖亭

这

总

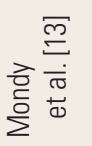

ย
产 $\frac{\infty}{2}$

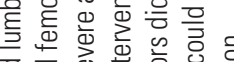

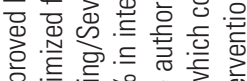

를.

卷

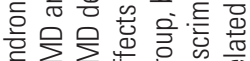

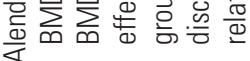

良 $\frac{1}{\bar{c}}$

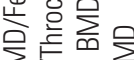

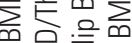

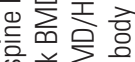

कि 힝

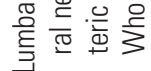

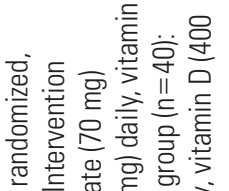

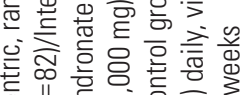

언

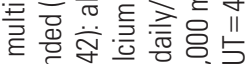

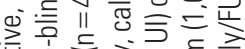

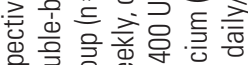

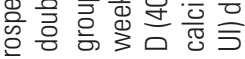

प웡

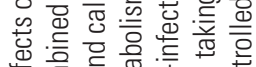

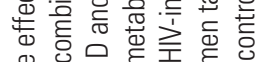

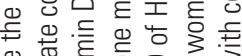

ه

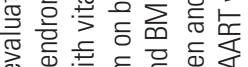

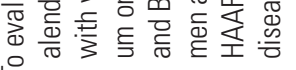

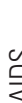

管

峞

空

홍

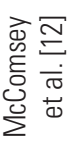

룽

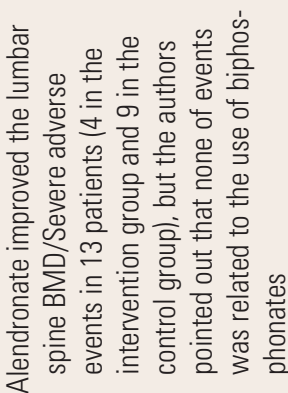

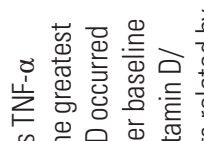

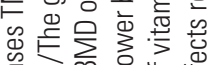

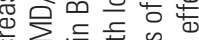

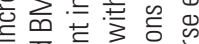

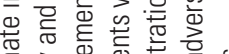

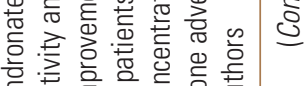

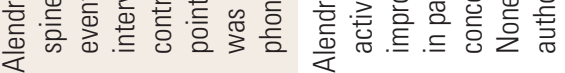

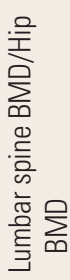

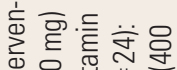

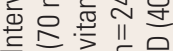

要离

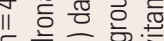

들 产要

है

응 을

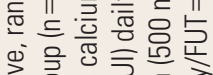

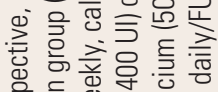

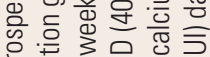

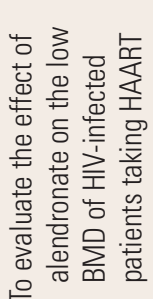

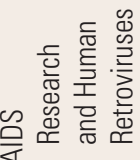

岩

总

ฐิ

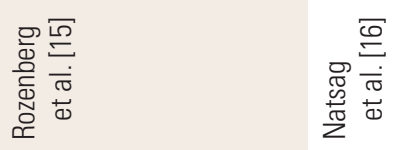

它

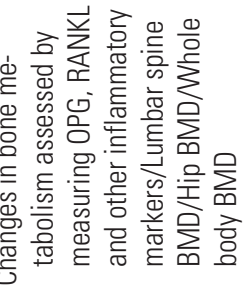

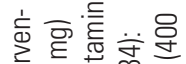

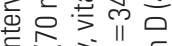

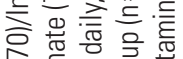

II 흔 $\overline{\mathrm{T}}$ 응

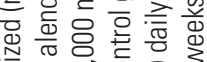

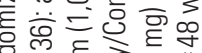

치에

힌

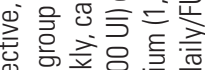

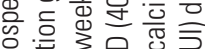

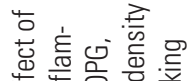

ब.

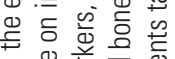

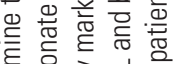

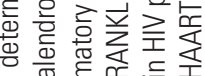

ㅇ

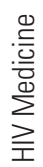

岩

变

空

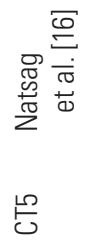




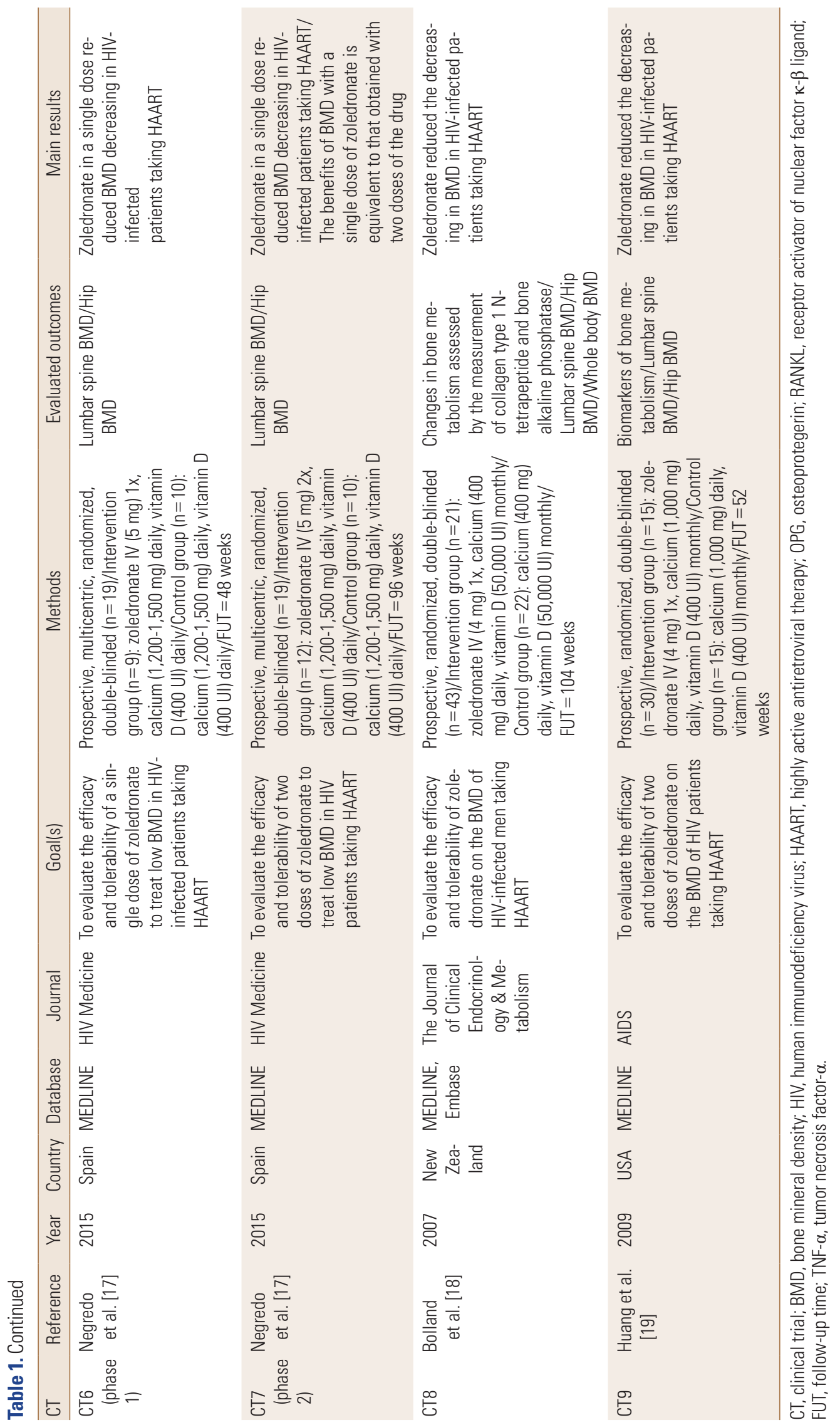


Table 2. Characteristics of global sample, intervention and control groups of meta-analysis

\begin{tabular}{|c|c|c|c|c|}
\hline Characteristics & Global sample & Intervention group & Control group & $P$-value ${ }^{\text {a) }}$ \\
\hline Total of patients $(k=9)$ & $394(100.0 \%)$ & $200(50.8 \%)$ & $194(49.2 \%)$ & $0.855^{\mathrm{bl}}$ \\
\hline Gender & & & & $0.815^{c)}$ \\
\hline Female & $73(18.5 \%)$ & $35(17.5 \%)$ & $38(19.6 \%)$ & \\
\hline Male & $321(81.5 \%)$ & $165(82.5 \%)$ & $156(80.4 \%)$ & \\
\hline Tobacco consumption $(k=6)$ & $198 / 300(66.0 \%)$ & $98 / 149(65.8 \%)$ & $100 / 151(66.2 \%)$ & 0.98 \\
\hline Alcohol consumption $(k=5)$ & $107 / 257(41.6 \%)$ & $53 / 128(41.4 \%)$ & $54 / 129(41.9 \%)$ & 0.877 \\
\hline Proportion of Caucasians $(k=6)$ & $256 / 300(85.3 \%)$ & $119 / 149(79.9 \%)$ & 137/151 (90.7\%) & 0.058 \\
\hline Biphosphonate used (k=9) & & & & $0.237^{c)}$ \\
\hline Alendronate (5 trials) & $268(68.0 \%)$ & $131(65.5 \%)$ & $137(70.6 \%)$ & \\
\hline Zoledronate (4 trials) & $126(32.0 \%)$ & $69(34.5 \%)$ & $57(29.4 \%)$ & \\
\hline Time of HIV infection (yr) (k=6) & $10.3 \pm 8.0$ & $10.3 \pm 6.2$ & $10.2 \pm 3.8$ & $0.982^{d)}$ \\
\hline Nadir of CD4+T cells (cells $/ \mu \mathrm{L})(\mathrm{k}=6)$ & $174.9 \pm 302.2$ & $174.3 \pm 159.9$ & $175.5 \pm 144.2$ & $0.730^{\mathrm{d})}$ \\
\hline CD4+T cells count (células/ $/ \mathrm{L}$ ) ( $\mathrm{k}=7$ ) & $491.50 \pm 434.4$ & $519.3 \pm 301.7$ & $462.38 \pm 334.7$ & $0.103^{\mathrm{d})}$ \\
\hline Lumbar spine T-score before intervention $(k=9)$ & $1.91 \pm 0.60$ & $1.97 \pm 0.72$ & $1.84 \pm 0.67$ & $0.055^{d l}$ \\
\hline Lumbar spine DMO before intervention $\left(\mathrm{g} / \mathrm{cm}^{2}\right)(\mathrm{k}=9)$ & $0.96 \pm 0.10$ & $0.97 \pm 0.10$ & $0.95 \pm 0.12$ & $0.098^{\mathrm{d})}$ \\
\hline
\end{tabular}

Quantitative variables are expressed by mean \pm standard deviation.

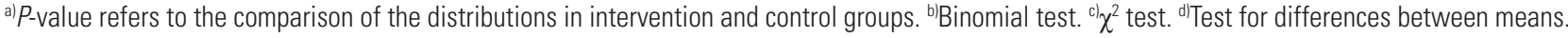
k, number of trials that registered the variable; HIV, human immunodeficiency virus; CD4, cluster of differentiation 4.
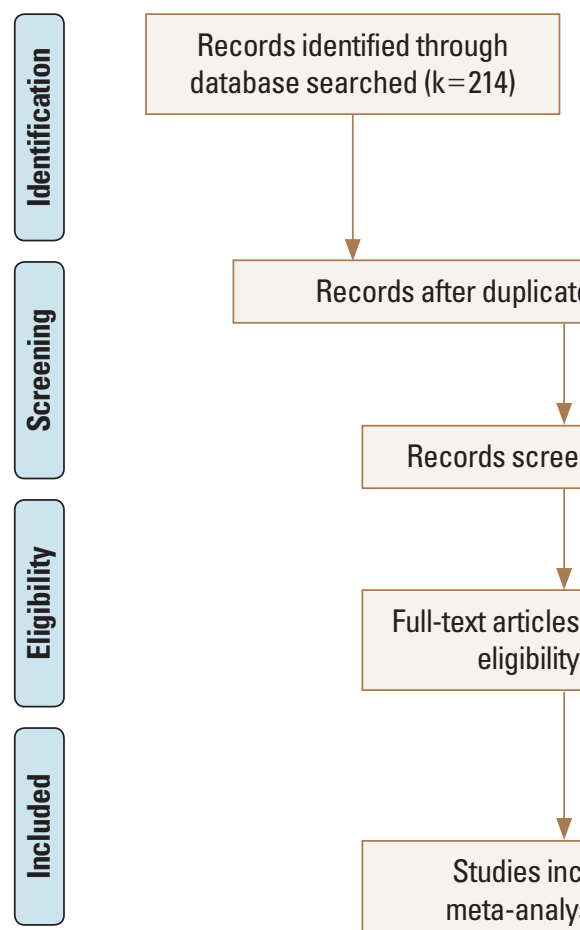

Additional records identified

through other sources $(k=0)$

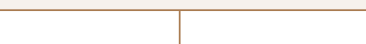

Records after duplicates removed $(\mathrm{k}=210)$

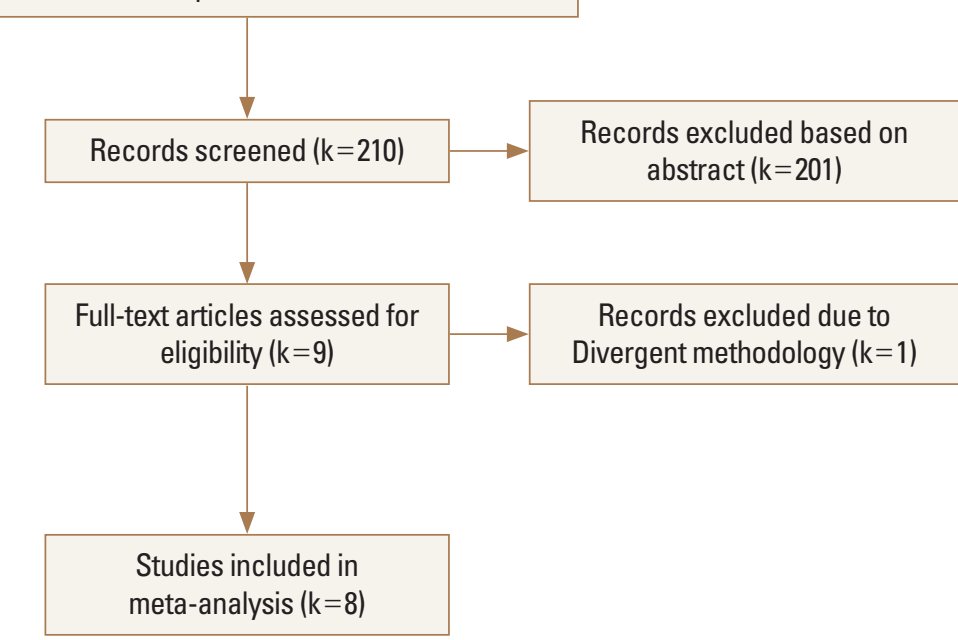

Fig. 1. Flowchart (Preferred Reporting Items for Systematic Reviews and Meta-Analysis [PRISMA flow]) for the identification and selection of trials for systematic review and meta-analysis. k, number of trials that registered the variable.

al selected, the data collected follows in Tables 1 and 2 .

Results were presented using an organization chart to studies selection and forest plots for the comprehension of the meta-analysis. We used Cochrane's $Q$ and $I^{2}$ statistics to address heterogeneity and consistency of trials (significant if $P \leq 0.05$ in $Q$ and $I^{2}$ greater than $50 \%$ ). The difference between the groups compared (intervention and control) was made at the significance level of $5 \%(a=0.05)$. 


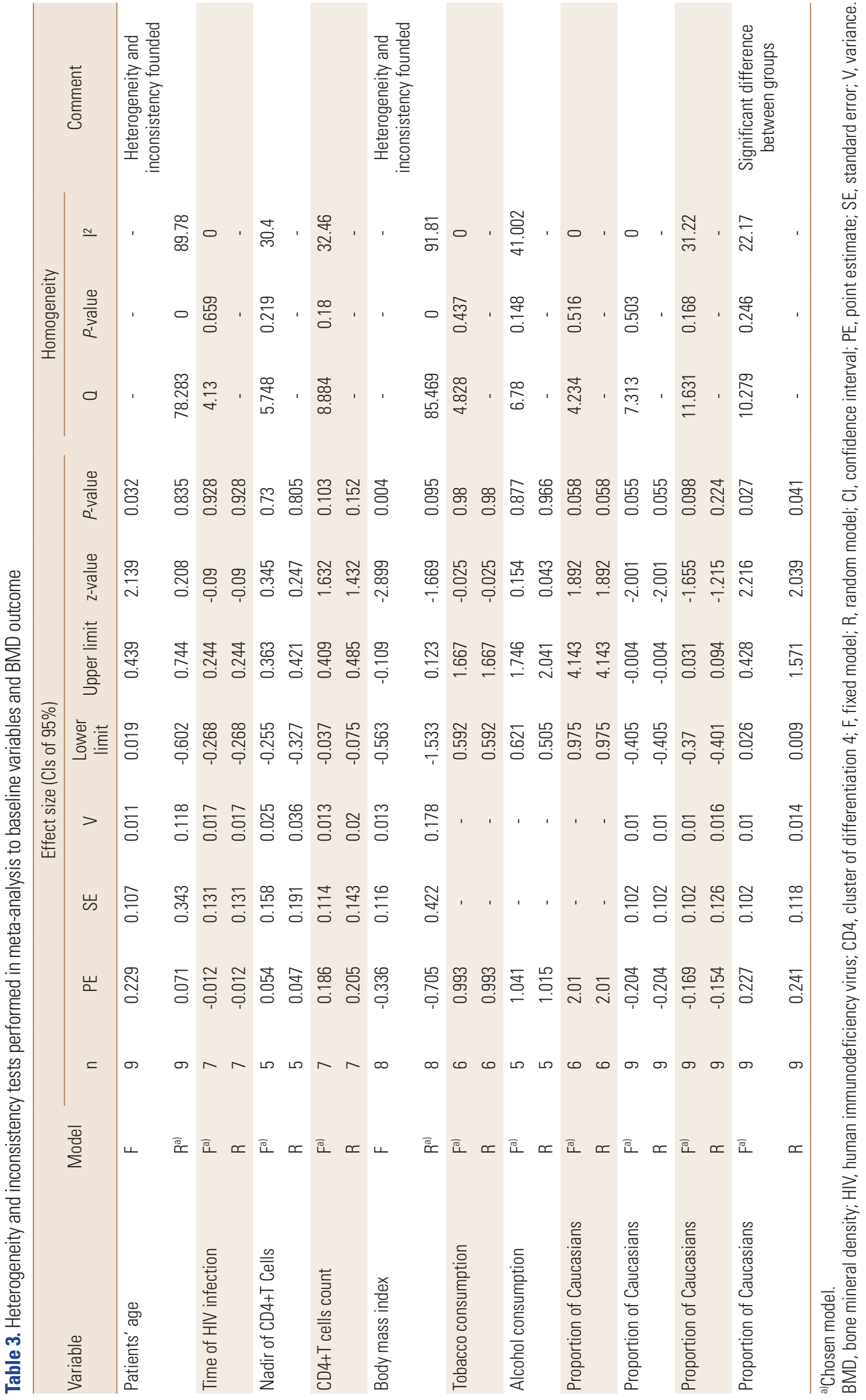




\begin{tabular}{|c|c|c|c|c|c|c|c|c|c|}
\hline & SMD & SE & v & LL & UL & $z$-value & p-value & & \\
\hline CT1. Guaraldi et al. [14] & -0.179 & 0.315 & 0.099 & -0.797 & 0.439 & -0.569 & 0.569 & & \\
\hline CT2. Mondv et al. [13] & 0.479 & 0.365 & 0.133 & -0.236 & 1.193 & 1.313 & 0.189 & & \\
\hline CT3. McComsey et al. [12] & 2] 0.086 & 0.220 & 0.049 & -0.347 & 0.519 & 0.389 & 0.697 & & \\
\hline CT4. Rozenberg et al. [15] & 0.633 & 0.310 & 0.096 & 0.026 & 1.241 & 2.042 & 0.041 & & \\
\hline CT5. Natsag et al. [16] & -0.048 & 0.239 & 0.057 & -0.517 & 0.421 & -0.202 & 0.840 & & \\
\hline CT6. Negredo et al. [17] & -0.109 & 0.384 & 0.148 & -0.863 & 0.644 & -0.284 & 0.777 & & \\
\hline CT7. Negredo et al. [17] & 0.483 & 0.434 & 0.189 & -0.368 & 1.334 & 1.112 & 0.266 & & \\
\hline CT8. Bolland et al. [18] & 0.823 & 0.318 & 0.101 & 0.200 & 1.446 & 2.590 & 0.010 & & \\
\hline CT9. Huang et al. [19] & 0.319 & 0.367 & 0.135 & -0.401 & 1.039 & 0.867 & 0.386 & & \\
\hline GLOBAL (fixed) & 0.227 & 0.102 & 0.010 & 0.026 & 0.428 & 2216 & 0.027 & & \\
\hline & & & & & & & & Control Gro & \\
\hline
\end{tabular}

Fig. 2. Forest plot of meta-analysis for lumbar spine bone mineral density after intervention. SMD, standardized mean differences; SE, standard error; V, variance; LL, lower limit; UL, upper limit.

Table 4. Means and standard deviations of lumbar spine BMD after intervention of the CTS

\begin{tabular}{llccc}
\hline CT & \multicolumn{1}{c}{ Reference } & Year & $\begin{array}{c}\text { Intervention } \\
\text { group }\end{array}$ & $\begin{array}{c}\text { Control } \\
\text { group }\end{array}$ \\
\hline CT1 & Guaraldi et al. [14] & 2004 & $0.97 \pm 0.11$ & $1.00 \pm 0.17$ \\
CT2 & Mondy et al. [13] & 2005 & $0.97 \pm 0.08$ & $0.92 \pm 0.12$ \\
CT3 & McComsey et al. [12] & 2007 & $0.94 \pm 0.07$ & $0.93 \pm 0.07$ \\
CT4 & Rozenberg et al. [15] & 2012 & $0.91 \pm 0.06$ & $0.87 \pm 0.08$ \\
CT5 & Natsag et al. [16] & 2016 & $0.91 \pm 0.13$ & $0.91 \pm 0.15$ \\
CT6 (phase 1) Negredo et al. [17] & 2015 & $0.98 \pm 0.10$ & $0.99 \pm 0.14$ \\
CT7 (phase 2) Negredo et al. [17] & 2015 & $1.04 \pm 0.04$ & $0.99 \pm 0.14$ \\
CT8 & Bolland et al. [18] & 2007 & $1.25 \pm 0.11$ & $1.14 \pm 0.16$ \\
CT9 & Huang et al. [19] & 2009 & $0.88 \pm 0.08$ & $0.86 \pm 0.08$ \\
& Weighted average & & $0.99 \pm 0.09$ & $0.95 \pm 0.12$ \\
\hline
\end{tabular}

$\mathrm{BMD}$, bone mineral density; CT, clinical trial.

For bias evaluation, we used the Bias Risk Assessment (BRA) tool recommended by the Cochrane Manual,[20] according to which the reviewers chose and classified the risk of bias to each source of bias and each trial in 4 categories: absent, low, medium, or high. Thus, to each trial and each source of bias was calculated the bias risk as the double of a weighted average of these categories frequencies (risk absent - weight 0; low risk - weight 1 ; medium risk weight 2; high risk - weight 3). BRA is a measure that varies from 0 to $100 \%$. The risk of publication bias was assessed using the graphical assessment of the funnel plot on BMD outcome.

Sensitivity analysis testing the influence of each study on the overall results was done by omitting one study at a time to explore potential sources of heterogeneity and to test the stability of regrouped results.

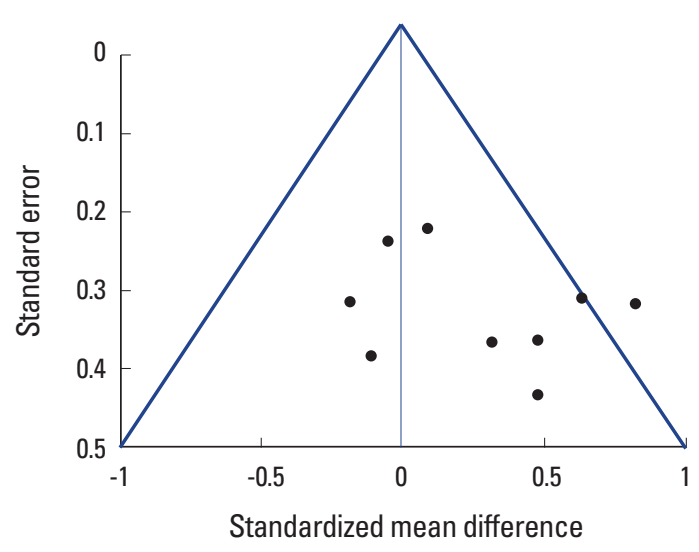

Fig. 3. Funnel plot of publication bias on bone mineral density outcomes.

Descriptive analyzes were performed using the IBM SPSS Statistics for Windows (version 20.0; IBM Corp., Armonk, NY, USA), and the software used for the meta-analysis was the Comprehensive Meta-Analysis for Windows (version 3.0; Biostat Software Inc., Englewood, NJ, USA).

\section{RESULTS}

Full search results are represented in the flow-chart of Figure 1 . Out of the 214 publications identified, 8 trials met the criteria selection and were selected to be critically evaluated in relation to authenticity, methodological quality, and the importance of the information ( $\mathrm{k}=0.92$ of concordance between the 2 reviewers). All of them were controlled and compared the effects of biphosphonates (alendronate or zoledronate) combined with vitamin $\mathrm{D}$ and calcium on 
Table 5. Bias risk assessment for each trial according to source

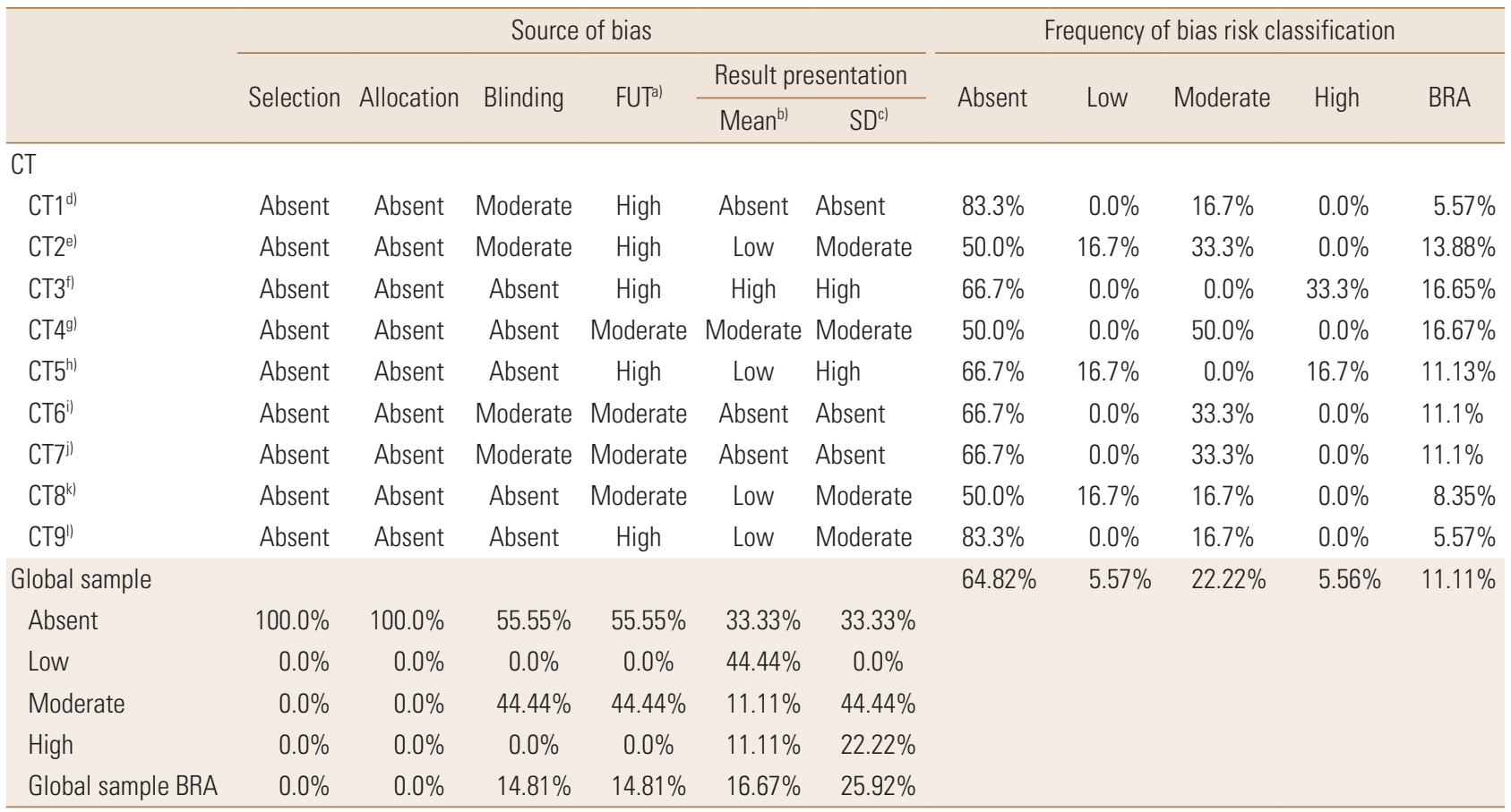

The BRA tool used was the one recommended by the Cochrane Manual [20].

CT, clinical trial; BRA, bias risk assessment; FUT, follow-up time; SD, standard deviation.

alFUTs differed significantly in CTs (48 to 104 weeks) and it was considered a possible source of bias. Applying BRA tool, we considered of 52 weeks of

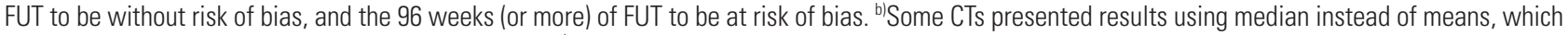
was considered a potential source of bias during BRA. ${ }^{c}$ Some CTs presented interquartile distances and range values instead of SD, which was considered

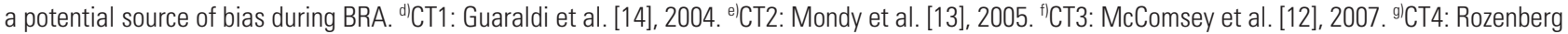
et al. [15], 2012. "'CT5: Natsag et al. [16], 2016. 'CT6: Negredo et al. [17], 2015. 'CT7: Negredo et al. [17], 2015. "'CT8: Bolland et al. [18], 2007. "CT9: Huang et al. [19], 2009.

HIV patients using HAART and presenting osteopenia or osteoporosis. Vitamin D and calcium was used in all trials by the control groups as a comparison parameter. Table 1 gathers the characteristics of the trials selected, as the bisphosphonates used in each of them, its dosage, and adverse effects related to use.

The trial by Negredo et al. [17] made an experiment with 2 different treatments over 2 years. In this review, we considered each treatment as an isolated trial to perform the meta-analysis (CT6 and CT7). The trial conducted by Pepe et al. [22] had a methodological approach different from the other trials, separating the treatment and control groups into other subgroups according to the patients' gonadal status. As none of the other trials had an approach like that used by Pepe et al. [22], this study was removed from the meta-analysis.

Random allocation of patients in groups was performed in all trials. The effects of intervention in bone metabolism were inferred by variables such as collagen $n$-tetra-peptide type 1, bone-specific alkaline phosphatase, osteoprotegerin, receptor activator of nuclear factor $\mathrm{k}-\beta$ ligand (RANKL), femoral neck BMD, LS BMD, femoral head BMD, whole-body $B M D$. As the measurement of $L S B M D\left(g / \mathrm{cm}^{2}\right)$ using bone densitometry was the unique variable assessed in all clinical trials, this was chosen as the main outcome of metaanalysis. The follow-up time (FUT) was quite discrepant among trials, ranging from 48 to 104 weeks (Table 1).

After gathering the participants of all trials, the global sample for meta-analysis had 394 patients, of whom 200 (50.8\%) received bisphosphonate, calcium, and vitamin D (intervention group); the other 194 patients (49.2\%) received only calcium and vitamin $D$ (control group). For these patients, the average of FUT was 62.9 weeks. The proportion of men was greater than women, 82.5 and $17.5 \%$, respectively; however, this disparity occurred in both study groups without statistical difference between the joint distribution of men and women in intervention and control groups. Characteristics of global sample, intervention and 
control groups of a meta-analysis are described in Table 2.

Table 3 summarizes the tests for heterogeneity and inconsistency performed with the model used in the analysis of SMD or, considering fixed or random effects for each variable. Meta-analysis for baselines variables showed no significant difference between groups before intervention (forest plots for baselines variables available as Supplementary Appendix 1); on the contrary, for the LS BMD after intervention (main outcome), a significant difference was found $(P=0.027)$ and the overall diamond does not cross value 0 (Fig. 2).

Most trials of meta-analysis have demonstrated some efficiency of bisphosphonates in reducing bone resorption and BMD increasing, however, some of them could not found statistical significance between the interventions and control groups. Table 4 shows the averages and SDs for LS BMDs after intervention.

The reviewers used and judged the possible sources of bias in the selected clinical trials: no random selection, no blinding, atypical FUT, absence of media, and SD of the variables in the papers, which were estimated by other biased statistics. Regarding the occurrence of bias, Table 5 shows the BRA for each trial and a global sample of metaanalysis. The Global BRA is $11.1 \%$, considering, in the scale from $0 \%$ to $100 \%$, refer to low bias. The possible impact of publication bias on BMD outcomes was explored by the funnel plot (Fig. 3). The studies were not evenly distributed across both sides of the funnel plot, reveling soft asymmetry. So, the visual inspection of funnel plots suggests that studies publishing negative effects may be missing. However, the Egger and Begg tests results suggest the absence of this source of bias ( $P=0.23$ for the Egger test and $P=0.33$ for the Begg test). Sensitivity analysis demonstrated that results remained statistically significant despite all deletions.

\section{DISCUSSION}

In the present meta-analysis, we found that the use of alendronate or zoledronate with calcium and vitamin $D$ could significantly improve the BMD in patients with HIV/ AIDS taking HAART (up to $0.227 \mathrm{~g} / \mathrm{cm}^{2}$ ). Considering the normal values of LS BMD $\left(1.237 \mathrm{~g} / \mathrm{cm}^{2}\right)$, the gain obtained with the intervention is sufficient to bring the average of LS BMD of patients with HIV/AIDS $\left(1.176 \mathrm{~g} / \mathrm{cm}^{2}\right)$ to the levels of not infected population, suggesting the existence of clinical benefits.[23]

The decrease in the risk of fractures due to bone frailty is certainly the main clinical benefit expected; however, establishing this correlation is a complex task that has been tried by other authors with minor success.[10,24-29] The hardest part of this correlation stems from the number of accumulated variables associated with the risk of fractures in HIV patients.[26,29] Obviously, since the reduction of BMD is an isolated risk factor for fractures in the non-infected population, we infer that the same should occur among HIV patients, although with a reduction in relative risk different from that observed in the general population. [30] Believing in this theoretical reduction in the risk of fractures when an increase in BMD is obtained, trials with HIV patients have been conducted investigating the role of vitamin $D$, calcium, and bisphosphonates for this purpose. As noted in previous reviews, the selected clinical trials did not allow us to establish a correlation between improved BMD and decreased risk of fracture.[27,31-34] The short FUT of patients was an important obstacle in this regard. As frailty fractures can occur at any time after the onset of osteoporosis, short-term trials do not allow us to establish this relationship, despite patients are using bisphosphonates or not.

Equally important regarding the use of bisphosphonates is the duration of its effects when its use is stopped. Studies with bisphosphonates in postmenopausal women suggest that patients with a t-score below -2.5 in the femoral neck still maintain a high risk of vertebral fractures even after 3 to 5 years of treatment, suggesting that prolonged use may be beneficial. Up to the time of writing this manuscript, the only study evaluating the prolonged use of a bisphosphonate (zoledronate) in patients with HIV/AIDS demonstrated that the effects of 2 annual doses lasted for five years after the second medication.[18] However, this study was relatively small and did not have enough strength to detect significant differences in fracture risk; the authors also recruited relatively young male patients with a t-score below -0.5 and with well-controlled disease, which makes it impossible to generalize their results to populations of patients with HIV/AIDS, low BMD and infection uncontrolled. In this meta-analysis, none of trials allowed us to assess adequately the effects of treatment interruption, nor the minimal time of use for an ideal outcome, either in terms of improving BMD or in the duration of the results obtained. 
In this meta-analysis the biphosphonates used were alendronate orally, and zoledronate intravenously. Both of them were, in general, well-tolerated. No effect related to its withdrawal was reported. The most common adverse effects related to alendronate use were gastrointestinal complaints, with similar frequencies in their occurrence in trials. Possibly, due to the longer use of alendronate in protocols for the treatment of osteoporosis, care in administering the drug is already widespread (avoiding the decubitus position after taking it and ingesting it with a large amount of liquid), reducing the occurrence of adverse effects. It was related to taking the medication. Considerations regarding the prolonged use of bisphosphonates, such as osteonecrosis of the jaw and atypical femoral fractures due to suppression of bone turnover, were not reported by the authors of the analyzed trials, possibly due to the rarity of these adverse events and the short duration of the trials analyzed. There is a greater hypothetical risk of adverse gastrointestinal events with the use of alendronate in relation to the use of zoledronate, especially with regard to the direct action of alendronate on the gastroesophageal mucosa, however, this risk cannot be assessed in the meta-analysis as it has not been properly presented for studies. Zoledronate, on the other hand, is used intravenously, being less frequently related to adverse gastrointestinal events, however, the occurrence of events related to the venous infusion is expected and was presented by trials that tested this drug. Regarding the route of administration, alendronate has the advantage of being able to be taken by patients themselves at home, while zoledronate needs hospitalization and a health team for its administration. In contrast, zoledronate has the dosage convenience when administered every six months, while alendronate is used weekly, which could be advantageous in patients taking several drugs, such as those used in HAART. Respecting the annual cost of treatment, zoledronate ( 2 annual doses) is more expensive than alendronate, hampering to use it on a large scale both for public health programs and for studies unlinked to the pharmaceutical industry that aim to measure its effectiveness and adverse effects.

The use of calcium and vitamin D was not homogeneous between studies, with calcium doses ranging from 400 to $1,500 \mathrm{mg} /$ day, and vitamin $D$ doses ranging from 400 to $800 \mathrm{IU} /$ day. Bolland et al. [18] used a monthly dose of $50,000 \mathrm{IU}$ of vitamin D ( $1.25 \mathrm{mg}$ of cholecalciferol). An ade- quate intake of vitamin D calcium is important for preventing bone demineralization and reducing the risk of fractures. The physiological needs of these nutrients vary throughout life, increasing with aging.[32,35,36] As vitamin D deficiency is prevalent in HIV patients, supplementation of this vitamin was used in association with calcium in both the treatment and control groups in all trials.[32] With regard to the doses of vitamin D used, previous studies carried out in patients who do not have HIV suggest a dose-dependent effect when demonstrating that supplementation with $400 \mathrm{IU} /$ day has less effect on the risk of fracture than its use in doses equal to or greater than $600 \mathrm{IU} /$ day. [37-39] Among HIV patients there is still no consensus in the literature regarding the dose required for supplementation and ideal serum levels of 1,25-hydroxy-vitamin D3 (25[OH]D3) for improving BMD and preventing frailty fractures.[40] Some authors consider that when the food intake of these elements is insufficient in HIV patients, Screening for hypovitaminosis should be done before starting the replacement of vitamin D and calcium.[40] In our review, only trials testing the effects of zoledronate considered serum levels of 1,25(OH)D3 as baseline characteristics among the study groups.[17-19,41]

Some risk factors for bone demineralization analyzed by the trials were included in the meta-analysis, such as age, white race, smoking, drinking, HIV infection time, indicators of the immune profile (nadir and CD4+ T cells), BMI and the $\mathrm{t}$-score of the LS before treatment. For these factors, there was no significant difference between the treatment and control groups in the meta-analysis sample. However, other risk factors for bone demineralization, such as physical activity, daily calcium intake, drugs used in HAART and serum calcium and vitamin D levels before interventions were not adequately presented by the authors and did not could be included in the meta-analysis, figuring as a significant limitation of this review. Other important limitations were the incomplete assessment of risk factors for bone loss among trials, the small number of trials, the short FUTs of the participants, and the methodological differences of study designs.

As some trials could not find by itself statistical differences between groups tested, despite all limitations above mentioned, the meta-analysis performed was relevant insofar as revealed no substantial heterogeneity, with high consistency and statistical significance to the main out- 
come considered. Consequently, as well as in the general population, the combination of Alendronate (orally) or zoledronate (intravenously), with calcium and vitamin D (orally) is effective to increase LS BMD in HIV patients. Further randomized clinical trials controlling both the variables related to bone demineralization and adverse effects can be useful to improve therapeutic protocols against bone loss in HIV.

\section{CONCLUSIONS}

This study provides enough evidence that BMD post-treatment is higher in HIV patients who used bisphosphonates combined with calcium and vitamin D.

\section{DECLARATIONS}

\section{Ethics approval and consent to participate Not applicable.}

\section{Conflict of Interest}

No potential conflict of interest relevant to this article was reported.

\section{ORCID}

Vinícius Magno da Rocha

https://orcid.org/0000-0001-5441-0679

Mariana Balardino Bogado Faria

https://orcid.org/0000-0002-9237-5664

Francisco de Assis dos Reis Júnior

https://orcid.org/0000-0003-0452-4525

Carla Ormundo Gonçalves Ximenes Lima

https://orcid.org/0000-0003-4649-5101

Rossano Kepler Alvim Fiorelli

https://orcid.org/0000-0001-5236-0903

Keila Mara Cassiano

https://orcid.org/0000-0002-5675-6953

\section{REFERENCES}

1. Hoy JF, Grund B, Roediger M, et al. Immediate initiation of antiretroviral therapy for HIV infection accelerates bone loss relative to deferring therapy: findings from the START bone mineral density substudy, a randomized trial. J Bone Miner Res 2017:32:1945-55.
2. Amorosa V, Tebas P. Bone disease and HIV infection. Clin Infect Dis 2006;42:108-14.

3. Saccomanno MF, Ammassari A. Bone disease in HIV infection. Clin Cases Miner Bone Metab 2011;8:33-6.

4. Althoff KN, Smit M, Reiss P, et al. HIV and ageing: improving quantity and quality of life. Curr Opin HIV AIDS 2016; 11:527-36.

5. Peck WA, Burckhardt P, Christiansen C, et al. Consensus development conference: diagnosis, prophylaxis, and treatment of osteoporosis. Am J Med 1993;94:646-50.

6. World Health Organization. WHO scientific group on the assessment of osteoporosis at primary health care level. Brussels, BE: World Health Organization; 2004.

7. Ofotokun I, Weitzmann MN. HIV-1 infection and antiretroviral therapies: risk factors for osteoporosis and bone fracture. Curr Opin Endocrinol Diabetes Obes 2010;17:523-9.

8. Dalla Grana E, Rigo F, Lanzafame M, et al. Relationship between vertebral fractures, bone mineral density, and osteometabolic profile in HIV and hepatitis B and C-infected patients treated with ART. Front Endocrinol (Lausanne) 2019;10:302.

9. Premaor MO, Compston JE. The hidden burden of fractures in people living with HIV. JBMR Plus 2018;2:247-56.

10. Sharma A, Shi $Q$, Hoover DR, et al. Frailty predicts fractures among women with and at-risk for HIV. AIDS 2019;33:45563.

11. Guaraldi G, Zona S, Menozzi M, et al. Cost of noninfectious comorbidities in patients with HIV. Clinicoecon Outcomes Res 2013;5:481-8.

12. McComsey GA, Kendall MA, Tebas $P$, et al. Alendronate with calcium and vitamin $D$ supplementation is safe and effective for the treatment of decreased bone mineral density in HIV. AIDS 2007;21:2473-82.

13. Mondy K, Powderly WG, Claxton SA, et al. Alendronate, vitamin $D$, and calcium for the treatment of osteopenia/osteoporosis associated with HIV infection. J Acquir Immune Defic Syndr 2005;38:426-31.

14. Guaraldi G, Orlando G, Madeddu G, et al. Alendronate reduces bone resorption in HIV-associated osteopenia/osteoporosis. HIV Clin Trials 2004;5:269-77.

15. Rozenberg S, Lanoy E, Bentata M, et al. Effect of alendronate on HIV-associated osteoporosis: a randomized, double-blind, placebo-controlled, 96-week trial (ANRS 120). AIDS Res Hum Retroviruses 2012;28:972-80.

16. Natsag J, Kendall MA, Sellmeyer DE, et al. Vitamin D, os- 
teoprotegerin/receptor activator of nuclear factor-kappaB ligand (OPG/RANKL) and inflammation with alendronate treatment in HIV-infected patients with reduced bone mineral density. HIV Med 2016;17:196-205.

17. Negredo E, Bonjoch A, Pérez-Álvarez N, et al. Comparison of two different strategies of treatment with zoledronate in HIV-infected patients with low bone mineral density: single dose versus two doses in 2 years. HIV Med 2015; 16: $441-8$

18. Bolland MJ, Grey AB, Horne AM, et al. Annual zoledronate increases bone density in highly active antiretroviral therapy-treated human immunodeficiency virus-infected men: a randomized controlled trial. J Clin Endocrinol Metab 2007; 92:1283-8.

19. Huang J, Meixner L, Fernandez S, et al. A double-blinded, randomized controlled trial of zoledronate therapy for HIVassociated osteopenia and osteoporosis. AIDS 2009;23:51-7.

20. Higgins JPT, Green S, editors. Cochrane handbook for systematic reviews of interventions version 5.1.0. London, UK: The Cochrane Collaboration; 2011.

21. Moher D, Liberati A, Tetzlaff J, et al. Items de referencia para publicar Revisiones Sistemáticas y Metaanálisis: La Declaración PRISMA. Rev Esp Nutr Hum Diet 2014;18:172-81.

22. Pepe J, Isidori $A M$, Falciano $M$, et al. Effect of risedronate in osteoporotic HIV males, according to gonadal status: a pilot study. Endocrine 2014;47:456-62.

23. Cotter AG, Sabin CA, Simelane S, et al. Relative contribution of HIV infection, demographics and body mass index to bone mineral density. AIDS 2014;28:2051-60.

24. Young B, Dao CN, Buchacz K, et al. Increased rates of bone fracture among HIV-infected persons in the HIV Outpatient Study (HOPS) compared with the US general population, 2000-2006. Clin Infect Dis 2011;52:1061-8.

25. Torti C, Mazziotti G, Soldini PA, et al. High prevalence of radiological vertebral fractures in HIV-infected males. Endocrine 2012;41:512-7.

26. Güerri-Fernandez R, Vestergaard P, Carbonell C, et al. HIV infection is strongly associated with hip fracture risk, independently of age, gender, and comorbidities: a population-based cohort study. J Bone Miner Res 2013;28:125963.

27. Shiau S, Broun EC, Arpadi SM, et al. Incident fractures in HIV-infected individuals: a systematic review and metaanalysis. AIDS 2013;27:1949-57.

28. Borderi M, Calza L, Colangeli V, et al. Prevalence of sub-clin- ical vertebral fractures in HIV-infected patients. New Microbiol 2014;37:25-32.

29. Yin MT, Shiau S, Rimland D, et al. Fracture prediction with modified-FRAX in older HIV-infected and uninfected men. J Acquir Immune Defic Syndr 2016;72:513-20.

30. Kanis JA, McCloskey $\mathrm{E}$, Johansson $\mathrm{H}$, et al. $\operatorname{FRAX}\left({ }^{\circledR}\right)$ with and without bone mineral density. Calcif Tissue Int 2012;90:113.

31. Brown TT. Challenges in the management of osteoporosis and vitamin D deficiency in HIV infection. Top Antivir Med 2013;21:115-8.

32. Castronuovo D, Cacopardo B, Pinzone MR, et al. Bone disease in the setting of HIV infection: update and review of the literature. Eur Rev Med Pharmacol Sci 2013;17:2413-9.

33. Reid IR, Bolland MJ, Grey A. Effects of vitamin D supplements on bone mineral density: a systematic review and meta-analysis. Lancet 2014;383:146-55.

34. Hileman CO, Eckard AR, McComsey GA. Bone loss in HIV: a contemporary review. Curr Opin Endocrinol Diabetes Obes 2015;22:446-51.

35. Gallagher JC. Vitamin D and aging. Endocrinol Metab Clin North Am 2013;42:319-32.

36. Curtis EM, Moon RJ, Dennison EM, et al. Prenatal calcium and vitamin D intake, and bone mass in later life. Curr Osteoporos Rep 2014;12:194-204.

37. Lips $P$, Graafmans WC, Ooms ME, et al. Vitamin D supplementation and fracture incidence in elderly persons. $A$ randomized, placebo-controlled clinical trial. Ann Intern Med 1996;124:400-6.

38. Meyer HE, Smedshaug GB, Kvaavik E, et al. Can vitamin D supplementation reduce the risk of fracture in the elderly? A randomized controlled trial. J Bone Miner Res 2002;17: 709-15.

39. Vieth R. Why the optimal requirement for Vitamin D3 is probably much higher than what is officially recommended for adults. J Steroid Biochem Mol Biol 2004;89-90:575-9.

40. Ryom L, Boesecke C, Gisler V, et al. Essentials from the 2015 European AIDS Clinical Society (EACS) guidelines for the treatment of adult HIV-positive persons. HIV Med 2016;17: 83-8.

41. Ofotokun I, Titanji K, Lahiri CD, et al. A single-dose zoledronic acid infusion prevents antiretroviral therapy-induced bone loss in treatment-naive HIV-infected patients: A phase Ilb trial. Clin Infect Dis 2016;63:663-71. 

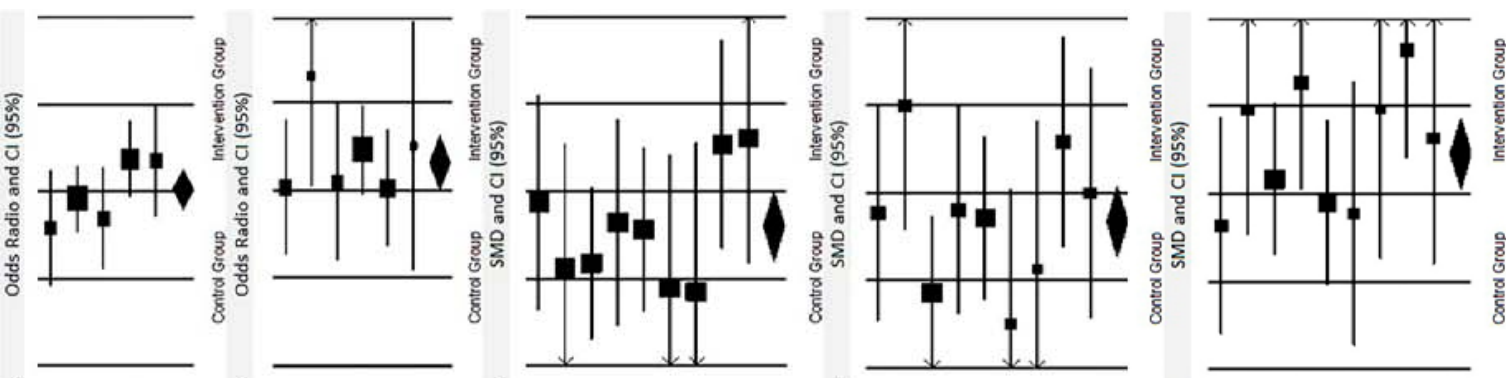

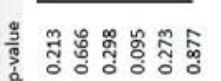

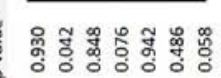

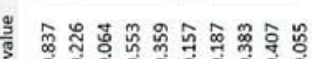

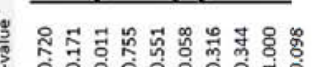

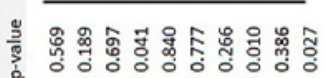

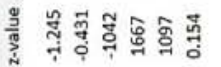

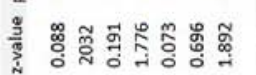

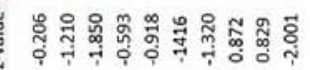

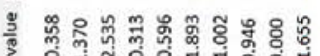

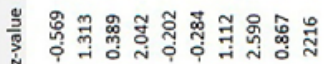

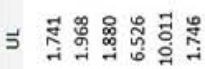

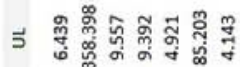

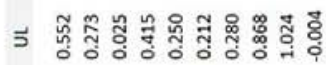

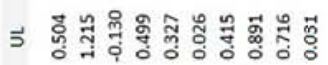

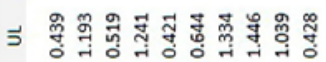

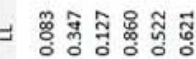

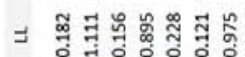

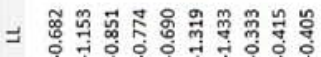

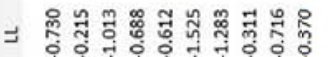

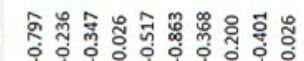

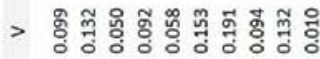

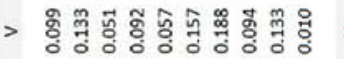

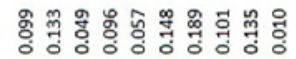

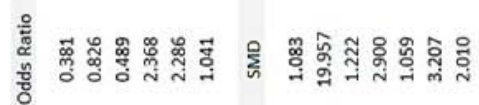

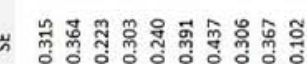

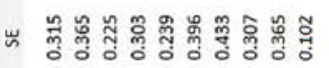

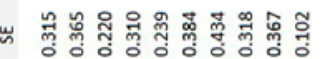

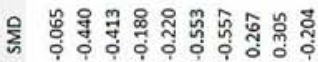

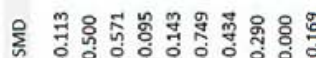

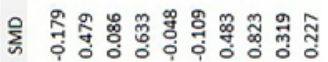

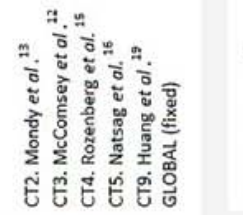

ws!|loyoo/y

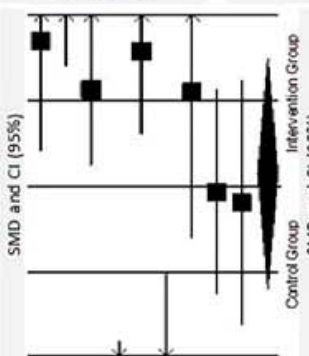

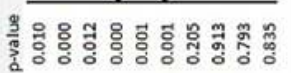

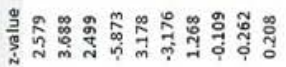

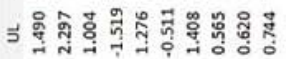

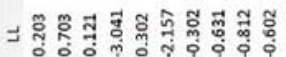

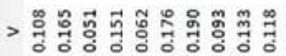

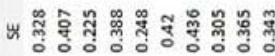

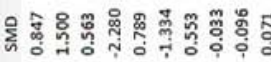

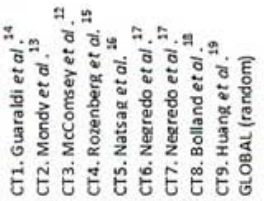

asy sivened

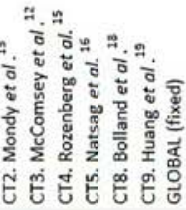

sueisesnes \%

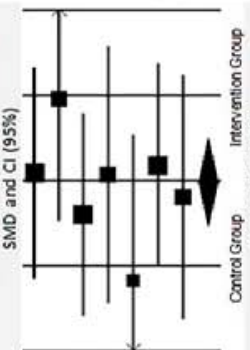

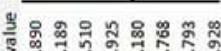

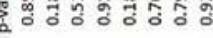

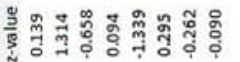
丂

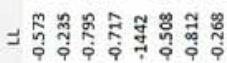

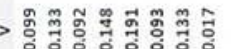

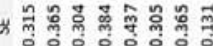

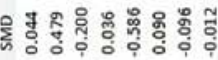

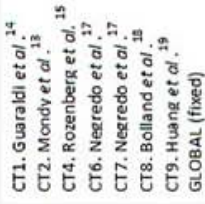

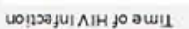

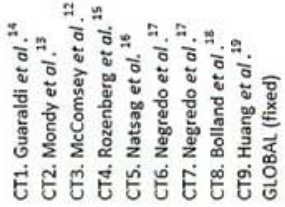

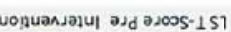

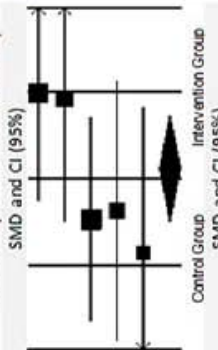

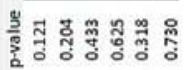

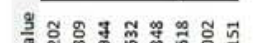

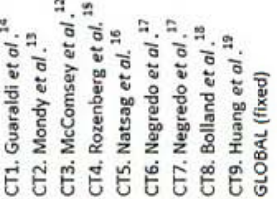

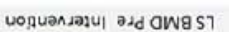

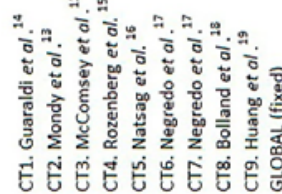

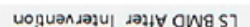
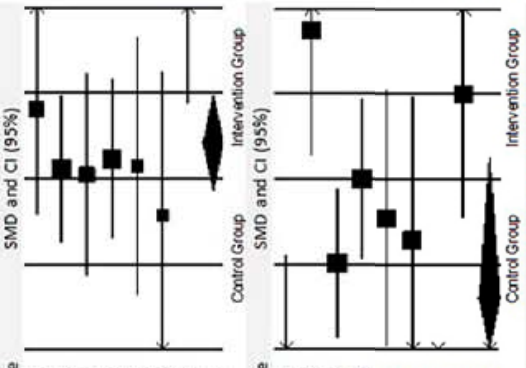

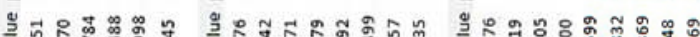

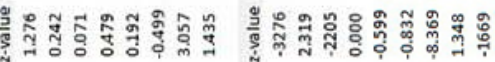

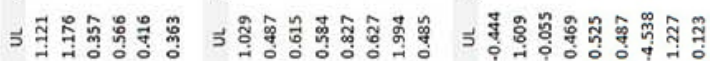

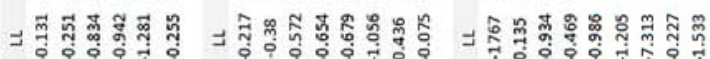

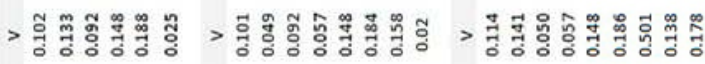

以

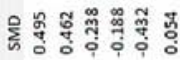

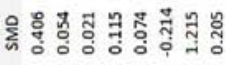

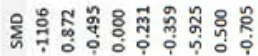
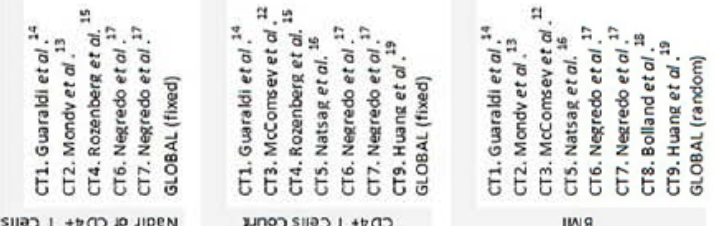

IN8

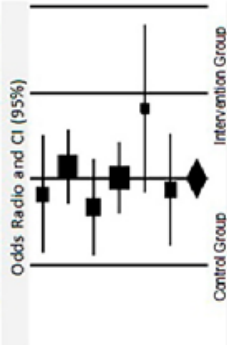

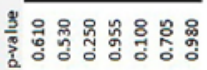

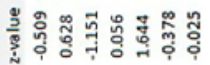

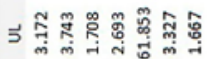

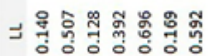

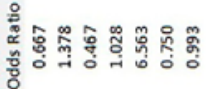

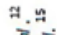

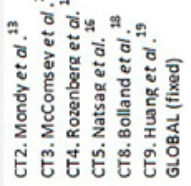

suryours

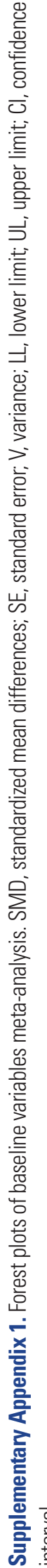


